\title{
Generation mechanism of diffuser stall in a centrifugal compressor with vaneless diffuser
}

\section{Original article}

Article history:

Submission date: 23 September 2020

Accepted date: 27 October 2020

Publication date: 4 December 2020

This is the updated version of a paper originally presented at the Global Power and Propulsion Virtual Technical Conference, GPPS Chania20, Sept. 7-9 2020.

\section{Check for updates}

*Correspondence:

NF: nobumichi-fuji@akane.waseda.jp

\section{Peer review:}

Single blind

\section{Copyright:}

(c) 2020 Fujisawa et al. @ This is an open access article distributed under the Creative Commons Attribution License (CC-BY 4.0), which permits unrestricted use, distribution, and reproduction in any medium, provided the original work is properly cited and its authors credited.

\section{Keywords:}

centrifugal compressor; vaneless diffuser; CFD; DES; rotating stall; internal flow

\section{Citation:}

Fujisawa N., Tajima K., Miida H., and Ohta Y. (2020). Generation mechanism of diffuser stall in a centrifugal compressor with vaneless diffuser. Journal of the Global Power and Propulsion Society. 4: 190-201. https://doi.org/10.33737/jgpps/128974

\author{
Nobumichi Fujisawa ${ }^{1,{ }^{*}}$, Kenta Tajima ${ }^{1}$, Hiroshi Miida ${ }^{1}, Y$ utaka Ohta ${ }^{1}$
}

${ }^{1}$ Waseda University, Tokyo, Japan

\begin{abstract}
The generation mechanism of a diffuser stall in a centrifugal compressor with a vaneless diffuser was experimentally and computationally investigated, and the main findings are presented in this paper. Diffuser stall is generated when the mass flow through the compressor decreases and approaches the compressor surge limit. It was observed that diffuser stall cells rotate at $25-30 \%$ of the impeller rotational speed, with diffuser stall fluctuations observed at $180^{\circ}$ from the cutoff (volute tongue). It was found that the magnitude of the diffuser stall fluctuation gradually increased near the cutoff. According to the CFD analysis, the mass flow fluctuations at the diffuser exit showed a low mass flow region, rotating at approximately $25 \%$ of the impeller rotational speed. The diffuser stall fluctuations began at $180^{\circ}$ from the cutoff and developed as this region approached the cutoff. Therefore, the diffuser stall could be simulated by CFD analysis. First, the diffuser stall cell originated at $180^{\circ}$ from the cutoff by the hub side boundary layer separation. Then, the diffuser stall cell further developed by the boundary layer separation accumulation and the induced low velocity area. The low velocity region formed a blockage across the diffuser passage span. The diffuser stall cell expanded due to the boundary layer separations occurring on the shroud and hub wall by turns. Finally, the diffuser stall cell vanished when it passed the cutoff, because the mass flow recovery occurred. The current paper presented the main results of this phenomena of great importance in centrifugal compressors.
\end{abstract}

\section{Introduction}

A centrifugal compressor is mostly used in a wide range of applications due to a high pressure ratio and small size. Centrifugal compressors with a vaneless diffuser are often employed due to their wider stability range, compared to those with a vaned diffuser. However, the operating range is limited by the onset of rotating stall and surge. Prior to the onset of the surge, the rotating stall was generally occurred. Therefore, to enhance the operating range and suppress the surge onset, one should must focus on understanding the rotating stall generation mechanism.

Numerous researchers have reported rotating stall behavior in centrifugal compressors with vaneless diffuser. The tornado-type separation vortex caused by full blade leading-edge separation was observed under the flow field of a developed rotating condition (Iwakiri et al., 2009). Zhang et al. investigated the volute influence on stall inception onset and development. Under the volute influence, the spillage vortices at a leading edge of impeller blades were developed by a static pressure peak near the tongue (Zhang et al., 2019). Therefore, the centrifugal compressor impeller stall was strongly related to a suction side separation and 
tip-leakage vortex. Additionally, many reports discuss a diffuser stall behavior in a vaneless diffuser. Senoo et al. were the first to investigate the relationship between reverse flow and the initiation of a vaneless diffuser rotating stall in a centrifugal blower. They proposed initiation criteria for a vaneless diffuser rotating stall (Senoo et al., 1977; Senoo and Kinoshita, 1978). Ohuchida et al. suggested both a boundary layer separation pattern and a stall propagation mechanism in a vaneless diffuser by PIV experiments (Ohuchida et al., 2013). They indicated that a rotating reverse flow disturbance in the diffuser was related to developing of a rotating stall cell, blocking interior diffuser flow at near-surge. However, the detailed diffuser stall generation mechanism in a vaneless diffuser was not completely understood.

Previous publications revealed the mechanism of rotating stall in the centrifugal compressor with vaned diffuser (Fujisawa and Ohta, 2017; Fujisawa et al., 2019a,b). The current study is focused on the generation mechanism of a diffuser stall in vaneless diffuser. Both experimental and numerical analysis were conducted on stall inception of centrifugal compressor with vaneless diffuser. The unsteady velocity was measured in circumferential and meridional direction within vaneless diffuser. Furthermore, Detached Eddy Simulation (DES) analysis over the entire compressor region, including the volute, was performed to reveal more detailed diffuser stall cell behavior.

\section{Experimental apparatus and procedure}

The tested centrifugal compressor schematic view for this study is shown in Figure 1. The test rig at Waseda University was used for a shipboard turbocharger, consisting of an impeller, vaneless diffuser, and volute. The impeller is equipped with seven full blades and seven splitter blades. The geometry details, as well as key parameters, of the tested compressor are summarized in Table 1. The impeller is an open-type impeller with a tip clearance that is $1.0 \%$ of the impeller blade width at $B_{1}=105.6 \mathrm{~mm}$. The impeller rotational speed was set to $6,000 \mathrm{~min}^{-1}$.

The steady pressure was measured at the compressor exit by a differential pressure transducer (Yamatake JTD920A). The orifice flow meter was set at the outlet duct to measure the steady mass flow rate. Velocity fluctuations, which defined as the difference between the time averaged value and the peak one, were measured on the test rig using a hot-wire anemometer (DANTEC, 55R57). The circumferential measurement points were placed at five positions $\left(0^{\circ}, 90^{\circ}, 150^{\circ}, 180^{\circ}\right.$ and $\left.240^{\circ}\right)$, respectively as shown in Figure 1 . The meridional velocity measurement points were at the diffuser inlet (D.I.), diffuser midsection (D.M.), and diffuser exit (D.E.). The hot-wire probes traversed in the spanwise direction in $5 \%$ increments of the diffuser width. The probe body is a $2.3 \mathrm{~mm}$ diameter, which is about $10 \%$ of the diffuser passage width. Although this probe size slightly influenced on the flow blockage, the scale of stall behavior would be greatly larger compared with the velocity fluctuation induced by the probe effect.

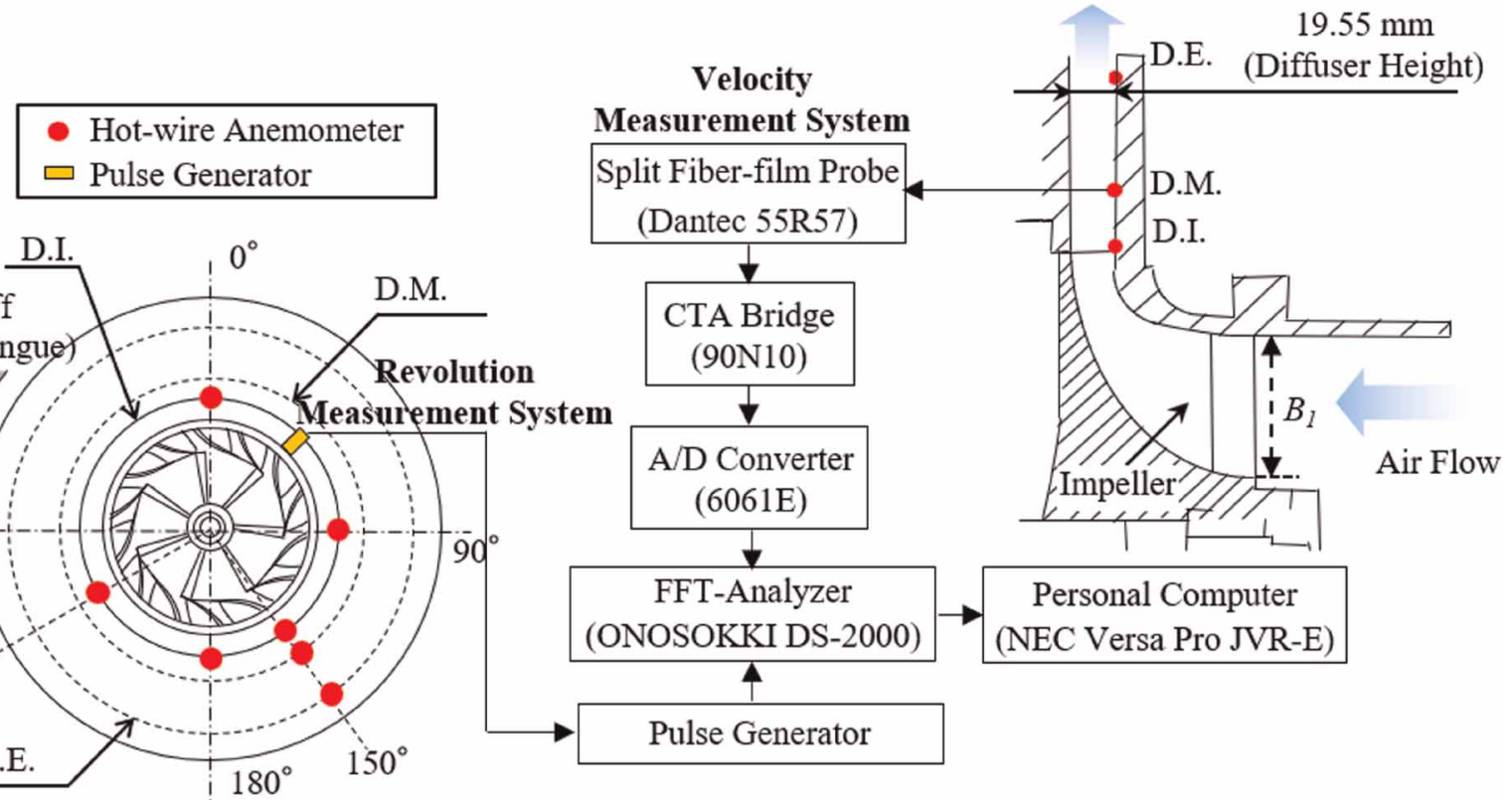

Figure 1. Experimental Apparatus and Procedure. 
Table 1. Dimensions of tested compressor.

\begin{tabular}{|c|c|c|c|c|c|}
\hline \multicolumn{3}{|l|}{ Tested Centrifugal Compressor } & \multicolumn{3}{|l|}{ Diffuser } \\
\hline Rotational Speed & $N$ & $6,000 \mathrm{~min}^{-1}$ & Inlet Diameter & $D_{3}$ & $328 \mathrm{~mm}$ \\
\hline Mass Flow Rate (design point) & $G$ & $1.64 \mathrm{~kg} / \mathrm{s}$ & Outlet Diameter & $D_{4}$ & $559 \mathrm{~mm}$ \\
\hline Pressure Ratio (design point) & $p_{5} / p_{1}$ & 1.1 & Diffuser Width & $B_{4}$ & $19.55 \mathrm{~mm}$ \\
\hline \multicolumn{6}{|l|}{ Impeller } \\
\hline Number of Blades & $Z$ & 14 & & & \\
\hline (Main + Splitter) & & $(7+7)$ & & & \\
\hline Inlet Diameter & $D_{1}$ & $248 \mathrm{~mm}$ & & & \\
\hline Outlet Diameter & $D_{2}$ & $328 \mathrm{~mm}$ & & & \\
\hline Exit Blade Width & $B_{2}$ & $26.14 \mathrm{~mm}$ & & & \\
\hline
\end{tabular}

\section{CFD methodology}

A detached eddy simulation (DES) code was developed for investigating the internal flow. The Computational Fluid Dynamics (CFD) code was constructed to solve the three-dimensional compressible Navier-Stokes equations. The convective flux was evaluated using the flux difference splitting (FDS) method and extended to the third-order MUSCL interpolation. The viscous flux was evaluated as a second-order central difference. The Matrix Free Gauss-Seidel (MFGS) implicit algorithm was employed for time integration. The shear stress transport (SST) k- $\omega$ turbulence model was used in this code and applied to DES. This model depends on the local turbulent length scale and grid spacing size. The dissipation term in the k-equation of the SST k- $\omega$ turbulence model is modified as follows:

$$
\begin{gathered}
D_{D E S}^{k}=\rho k^{3 / 2} / \widetilde{l} \\
\widetilde{l}=\min \left(l_{k-\omega}, C_{D E S} \Delta\right)
\end{gathered}
$$

where $l$ is the turbulent length scale, $\Delta$ is the local grid spacing, and the model's constant coefficient $C_{D E S}$ was set to the recommended value by the report (Strelets, 2001). The several studies reported that DES analysis is more suitable than URANS for detailed flow field investigations near stall in both axial and centrifugal compressor (Im et al., 2012; Yamada et al., 2017). Our In-house code was successfully validated for unsteady flow fields of various turbomachinery flows (Fujisawa et al., 2019b; Zhang et al., 2020).

The computational domains applied in the numerical simulations are illustrated in Figure 2. The grid system included 14 impeller passages, vaneless diffuser, and a volute. The impeller (including clearance region) and vaneless diffuser systems had 32.0 and 11.0 million cells, respectively. The volute region had 22.0 million cells. In total, the computational grid had 65.0 million cells. The wall cell width was $0.1 \mu \mathrm{m}$, corresponding to a y+ parameter, equal to approximately one along all solid surfaces. At the inflow boundary, the total pressure and temperature were fixed, while mass flow rates were fixed at the outflow boundary. Across a sliding boundary separating the moving impeller and stationary diffuser frames, the most recent data on one side were interpolated to obtain opposite side data by using an unsteady simulation sliding mesh. Nonslip and adiabatic conditions were adopted for the wall conditions. The main CFD setup was summarized in Table 2.

\section{Compressor performance and stall characteristics}

The experimental and numerical compressor performance results are shown in Figure 3. The numerical total pressure-rise characteristics were obtained from the time-averaged results of unsteady DES analysis and steady 

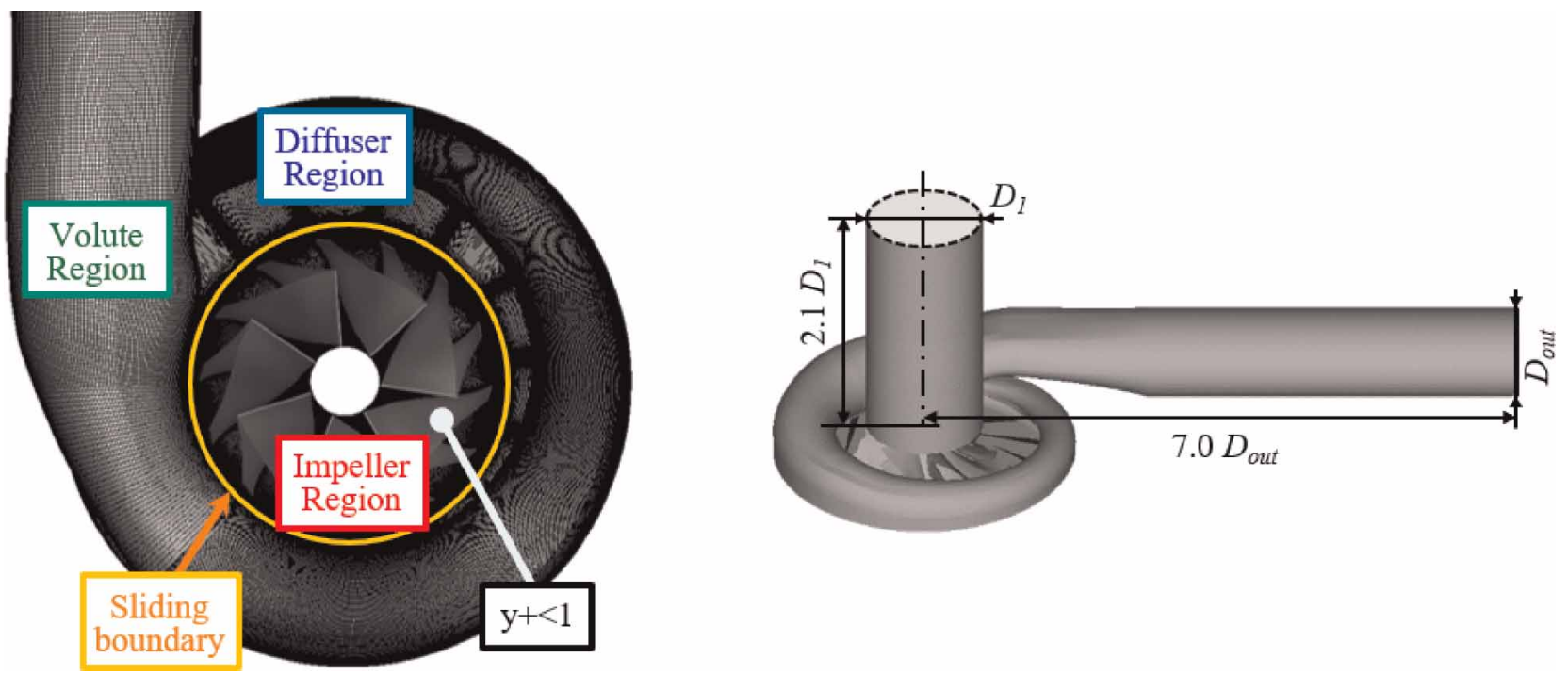

Figure 2. Numerical Mesh.

Table 2. Computational setup.

\begin{tabular}{|c|c|}
\hline \multicolumn{2}{|l|}{ Numerical Scheme } \\
\hline Convective Term & FDS (MUSCL 3rd-order Interpolation) \\
\hline Viscous Term & 2nd-order Central Differential Method \\
\hline Time Integral & MFGS \\
\hline Turbulence Model & SST k- $\omega$ Turbulence Model (DES) \\
\hline \multicolumn{2}{|l|}{ Boundary Conditions } \\
\hline Inlet Boundary & Total Pressure and Temperature Fixed \\
\hline Outlet Boundary & Mass Flow Rate Fixed \\
\hline RS Boundary & Sliding Mesh \\
\hline Time Steps & 5,000 step/rev \\
\hline \multicolumn{2}{|l|}{ Mesh } \\
\hline Impeller Region & 32.0 million cells \\
\hline Diffuser Region & 11.0 million cells \\
\hline Volute Region & 22.0 million cells \\
\hline Wall y+ & $<1$ \\
\hline
\end{tabular}




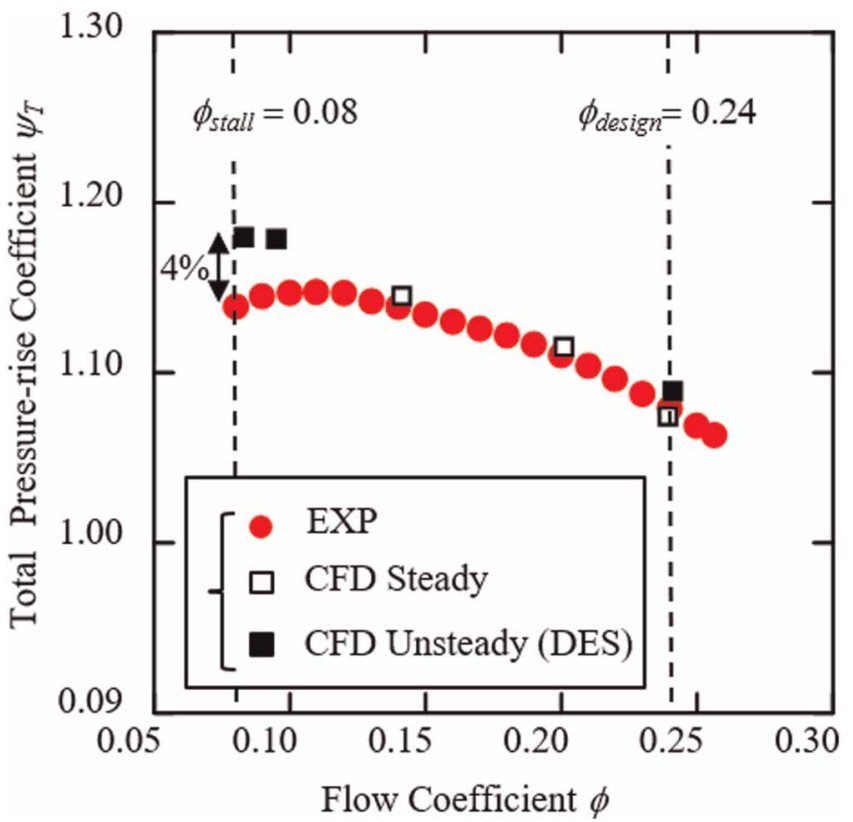

Figure 3. Compressor Performance.

RANS analyses. The flow and total pressure-rise coefficients are defined as follows:

$$
\begin{aligned}
\phi & =\frac{Q}{\pi^{2} D_{2}^{2} B_{2}(N / 60)} \\
\Psi_{t} & =\frac{\Delta p_{t}}{\rho \pi^{2} D_{2}^{2}(N / 60)^{2} / 2}
\end{aligned}
$$

The unsteady simulations were conducted at the operational design point $(\phi=0.24)$, and two off-design points $(\phi=0.10$ and 0.08$)$. The steady simulations were conducted at three operational points from $\phi=0.24$ to $\phi=0.14$. The steady RANS analysis results were in good agreement with measured results. The unsteady CFD results were overestimated particularly at the off-design points as compared with the test results. The mixing loss especially within scroll passages was assumed to be underpredicted at near stall point, but the main factor of overprediction of pressure rise coefficient still was not confirmed. However, the relative error between experimental and unsteady CFD results at off design points was within 5\%, as shown in Figure 3. Therefore, it was no problem to investigate the characteristics of a diffuser stall because the relative error was small.

To investigate the unsteady rotating stall characteristics, the radial velocity fluctuation of impeller discharge flow was measured with a split-film anemometer at $\phi=0.08$. The radial velocity was measured at D.I.0, 90, 180 and 240, which were indicated in Figure 1. The fast Fourier transformation (FFT) spatial distribution results at each measurement point in the spanwise direction are shown in Figure 4. The vertical axis represents the radial velocity fluctuation magnitude. From Figure 4, two types of velocity fluctuations were observed. One occurred at around $58 \mathrm{~Hz}$ and the other occurred at around $25-30 \mathrm{~Hz}$. Based on previous report (Fujisawa et al., 2016), the unsteady phenomena at around $58 \mathrm{~Hz}$ were caused by a rotating stall within the impeller passages. On the other hand, the lower frequency fluctuation at around $25-30 \mathrm{~Hz}$, was equivalent to $25-30 \%$ of the impeller rotating speed, and was caused by a rotating stall within the vaneless diffuser. Particularly, focusing on the diffuser stall, its characteristics varied depending on the circumferential position. From Figure 4(i), the small velocity fluctuation magnitudes caused by diffuser stall were observed near the shroud wall at D.I.0. Whereas, from Figure 4(ii), the large velocity fluctuation magnitude around $28 \mathrm{~Hz}$ was observed near the hub wall at D.I.90. Furthermore, Figure 4(iii) and (iv) show that the largest velocity fluctuation magnitude caused by the diffuser stall was observed near both shroud and hub walls at D.I.180 and 240, located near the cutoff. The mass flow fluctuations at the diffuser exit obtained from CFD analysis are shown in Figure 5. The red colored line indicated low mass flow regions and this rotational speed was about $25 \%$ of the impeller rotation speed. Therefore, the red colored low mass flow regions was assumed to be caused by the rotating diffuser stall, which rotated at 25\% of the impeller rotation speed. This low mass flow region appeared at $90^{\circ}$ and disappeared after passing the cutoff. 

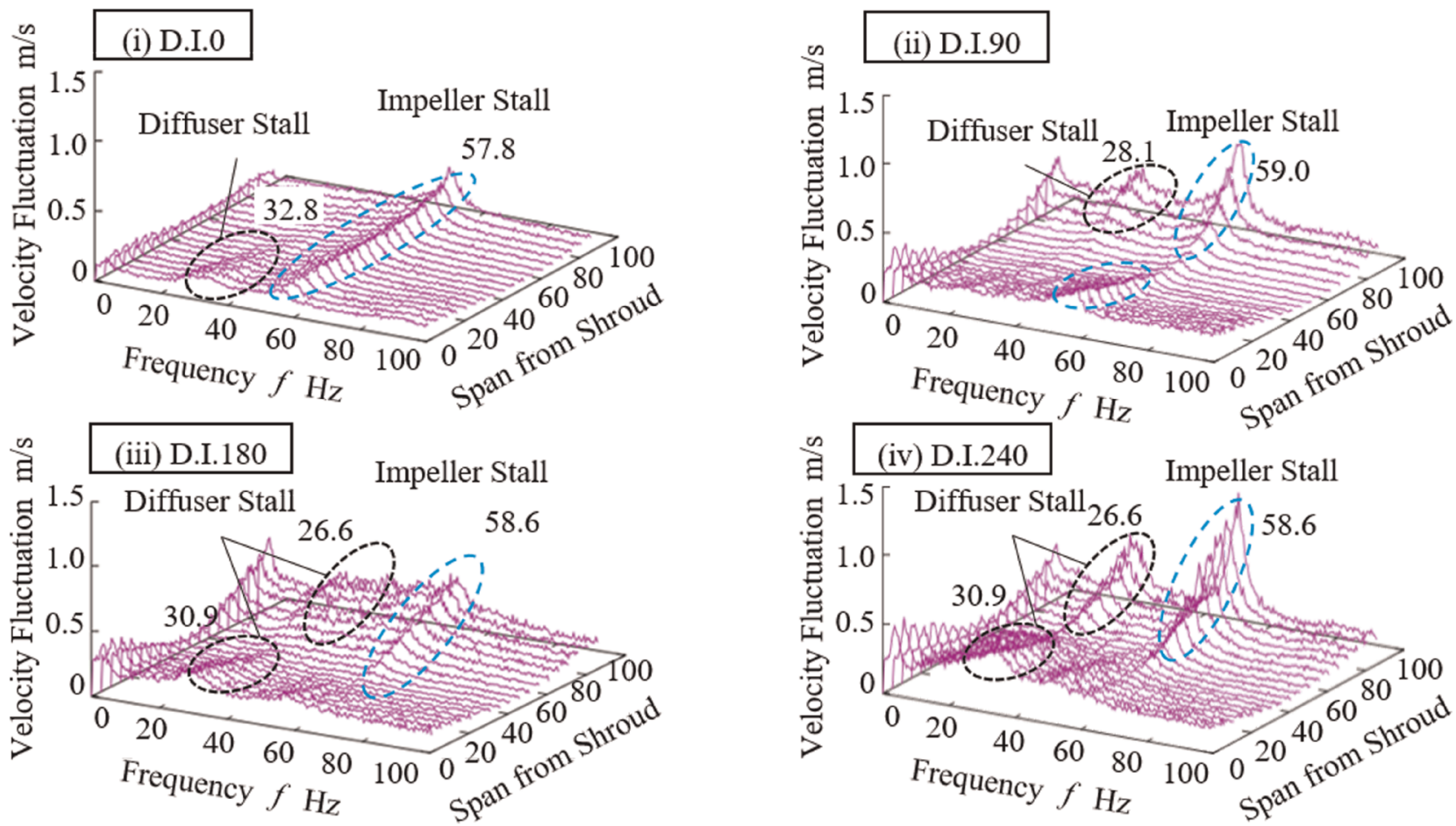

Figure 4. Distributions of Velocity Fluctuation in Spanwise Direction at Diffuser Inlet (EXP: $\boldsymbol{\phi}=0.08$ ).

Both experiment and CFD analysis indicated that the diffuser rotating stall initiated at $90^{\circ}$ and developed further as the diffuser stall cell approached the cutoff. After passing the cutoff, the diffuser rotating stall was attenuated. The detailed unsteady diffuser stall behavior is discussed later.

\section{Generation mechanism of diffuser stall}

To investigate the detailed unsteady diffuser stall behavior, an unsteady DES analysis was conducted. First, to understand the flow field, Figure 6 shows the distributions of radial velocity fluctuation within the diffuser passage obtained by CFD analysis. The radial velocity fluctuation was defined as follows:

$$
\Delta V_{r}(t)=V_{r}(t)-V_{r_{-} \text {ave }}
$$

$V_{r_{\text {ave }}}$ indicates the time-averaged radial velocity. The red color indicates the large magnitudes of radial velocity fluctuations. The region indicated by the white dashed circle is the diffuser stall cell that induced the largest mass flow fluctuations in Figure 5. Furthermore, the time-averaged mass flow rate of impeller discharge flow obtained from CFD analysis at $\phi=0.08$ is shown in Figure 7. The impeller exit plane mass flow rate was calculated on sections obtained by dividing the diffuser inlet into 32 circumferential sections. At $t^{*}=2.2$, the region with the large velocity fluctuations, as shown in Figure 6, was first generated at around $45^{\circ}$, where a strong circumferential

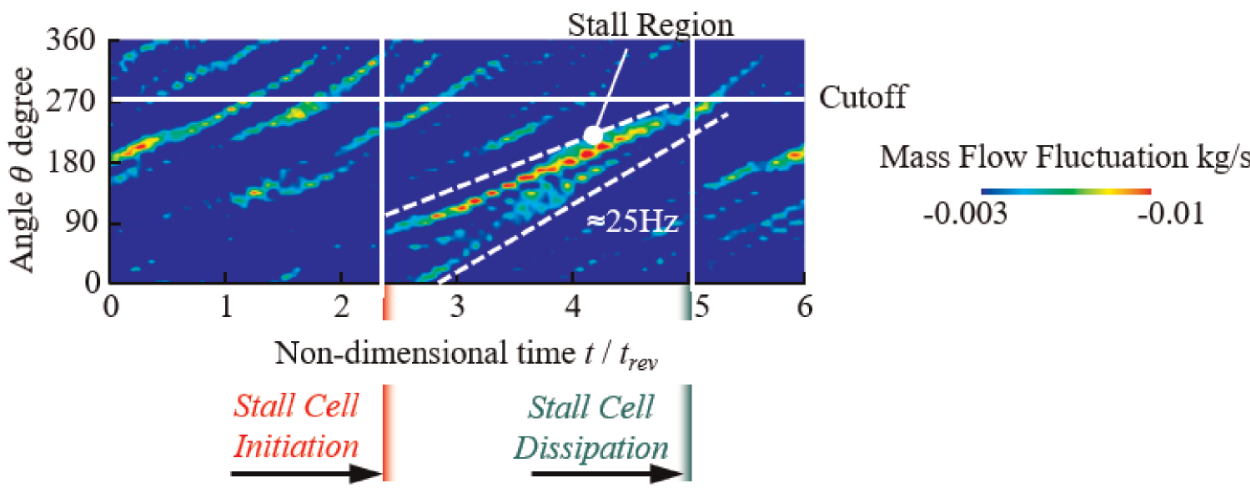

Figure 5. Mass Flow Fluctuation at Diffuser Exit (CFD: $\phi=0.08$ ). 


\section{Radial Velocity Fluctuation $\Delta V_{r} \mathrm{~m} / \mathrm{s}$}

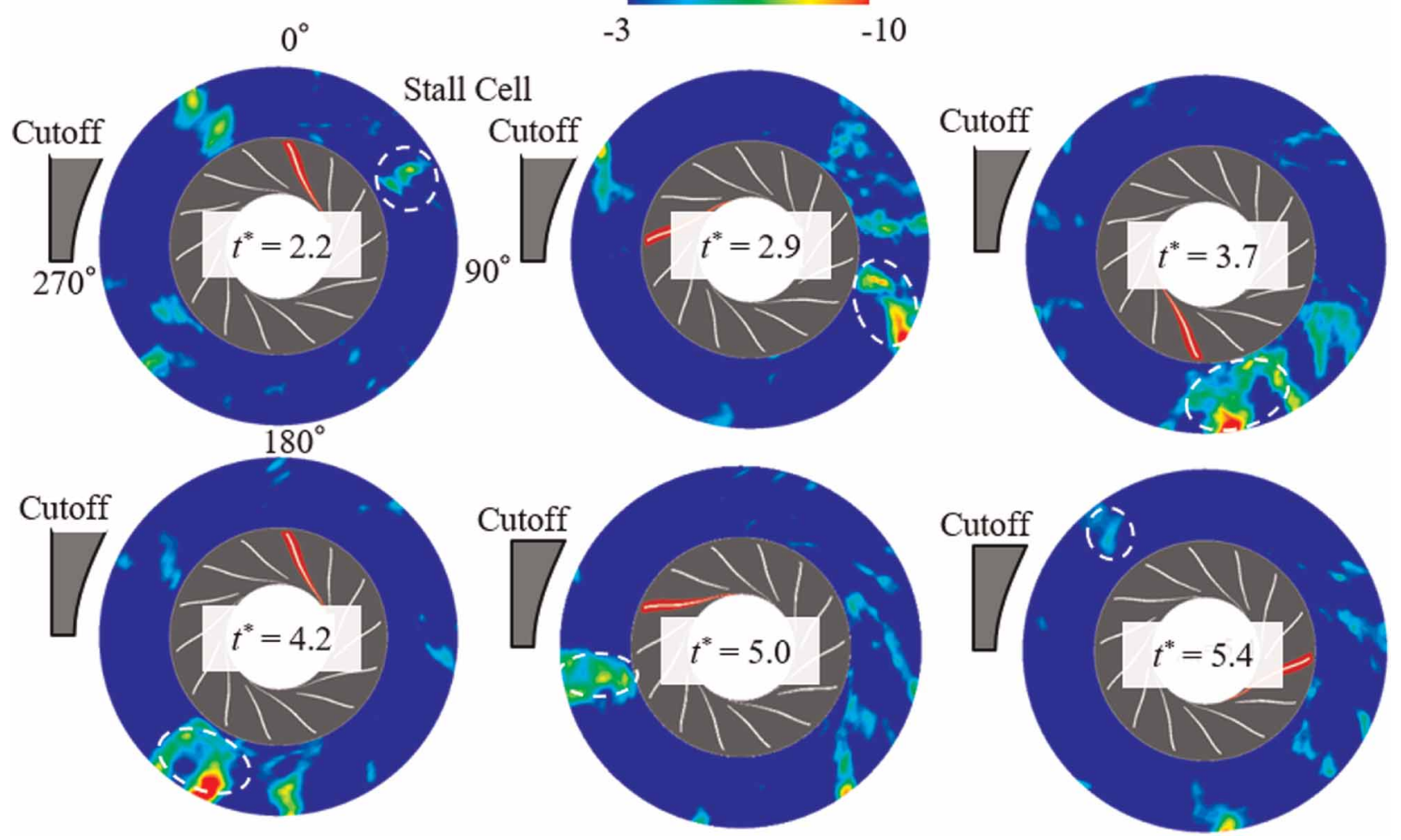

Figure 6. Distribution of Radial Velocity Fluctuations (CFD: $\boldsymbol{\phi}=0.08$ ).

adverse pressure gradient is observed, as depicted in Figure 8. Then, the large fluctuations region was formed at the diffuser exit area by the accumulation of several small backflow regions at $t^{*}=2.9$. After that, the diffuser stall cell expanded both radially and circumferentially as it approached the cutoff at $t^{*}=3.7$ and 4.2. Finally, the stall cell size reduced after passing the cutoff at $t^{*}=5.4$ due to increased impeller discharge mass flow rate at near the cutoff, as shown in Figure 7.

The circumferential static pressure distributions at $\phi=0.24$ and $\phi=0.08$ obtained from the CFD analysis are shown in Figure 8. The static pressure was measured at shroud wall D.M. From this figure, at around $300^{\circ}$, the static pressure at off-design point $\phi=0.08$ was the same level as that at design point $\phi=0.24$. However, from $0^{\circ}$ to the cutoff, the static pressure at $\phi=0.08$ was higher than that of $\phi=0.24$. The magnitude of circumferential adverse pressure gradient was strong near $0^{\circ}$ for $\phi=0.08$. Therefore, the diffuser stall was initiated at around $45^{\circ}$. Furthermore, the time-averaged meridional distribution of radial velocity and streamlines at $0^{\circ}, 45^{\circ}$, and $240^{\circ}$ are shown in Figure 9. The boundary layer separation occurred at hub and shroud wall by turns at 240deg, with numerous papers reporting the same phenomena (Van den Braembussche, 2019). From this paper, the spanwise pressure gradient induced the flow concentration on the side with the highest pressure. In addition, the spanwise pressure gradient inversion was generated once separation occurred, and this pressure gradient change continued until flow reversal took place on the other side. Therefore, the separations generated at both hub and shroud by turns. First, the flow concentrated on the shroud side where the total pressure was highest and boundary layer

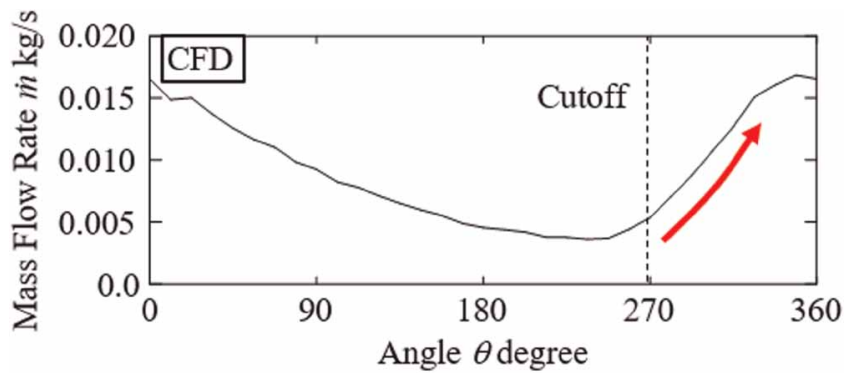

Figure 7. Distribution of Mass Flow at Impeller Exit (CFD: $\boldsymbol{\phi}=0.08$ ). 


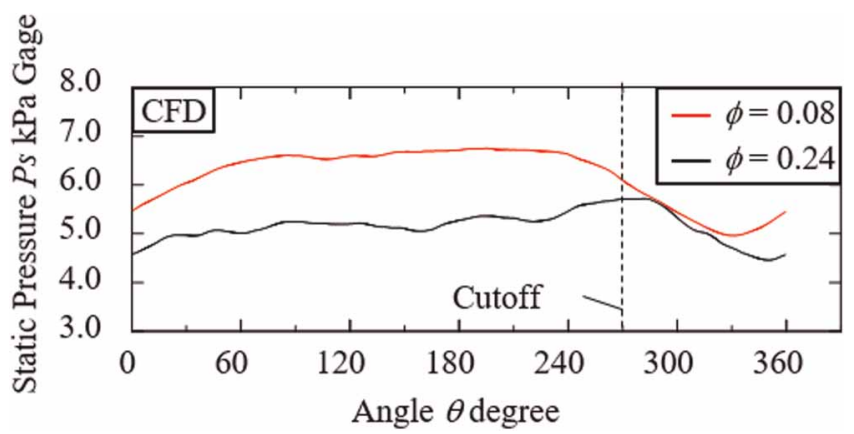

Figure 8. Distribution of Static Pressure in Circumferential Direction (CFD: $\boldsymbol{\phi}=0.24$ and 0.08 ).

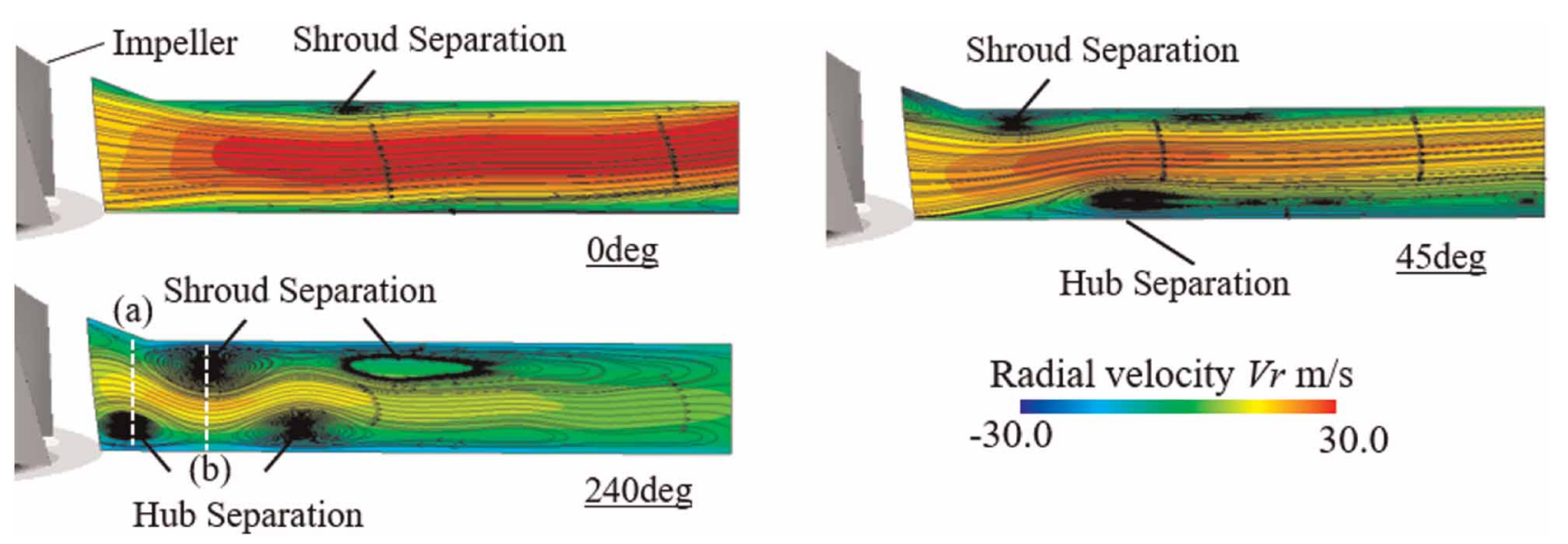

Figure 9. Meridional Distribution of Radial Velocity and Streamlines (CFD: $\boldsymbol{\phi}=0.08$ ).

separation occurred on the hub side at 240deg (point (a)), as shown in Figure 9. At point (b), the pressure gradient inversion occurred and the flow concentrated on the hub side. Therefore, the next boundary layer separation occurred at the opposite wall. Particularly, at $45 \mathrm{deg}$, the separation region was observed near the diffuser inlet shroud wall. Accordingly, the hub side boundary layer separations at around $45^{\circ}$, triggering the diffuser stall cell generation, were caused by the shroud side separation region at the diffuser inlet and a large circumferential adverse pressure gradient. Figure 10 shows the time-averaged limiting streamlines on hub wall obtained by measurement and CFD analysis. In the experiment, the limiting streamlines were visualized by using an oil film method. The limiting hub wall streamlines were bent at around $45^{\circ}$ where a small backflow region generated.

For improved understanding of the diffuser stall cell generation mechanism, the unsteady flow field was visualized. The instantaneous flow field at two different times are shown in Figure 11. The distributions of radial velocity fluctuation within the diffuser passage at each time are shown. The developing behavior of diffuser stall cell
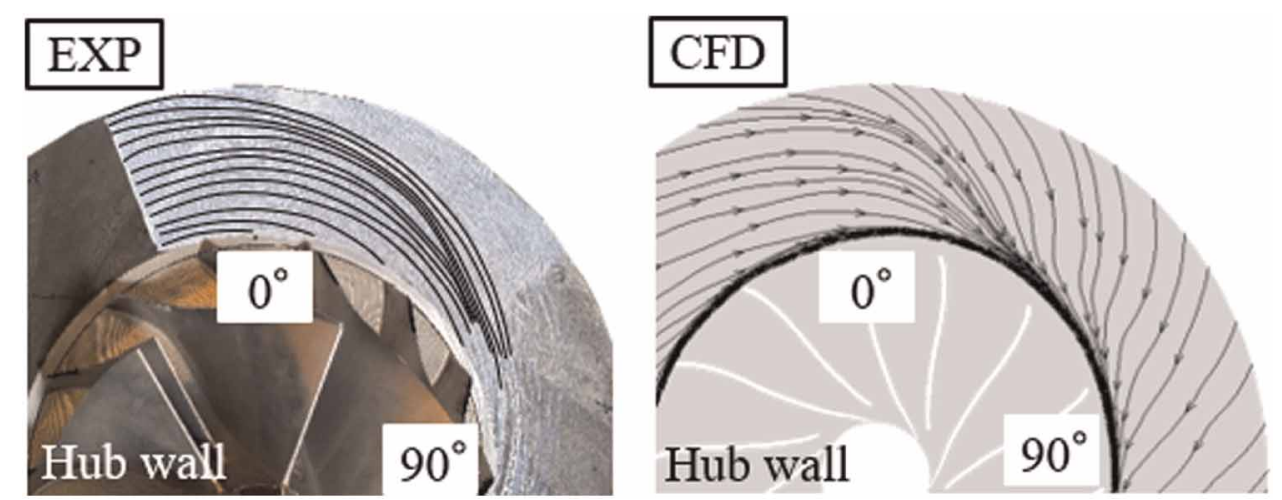

Figure 10. Limiting streamlines on hub wall (EXP and CFD: $\phi=0.08$ ). 


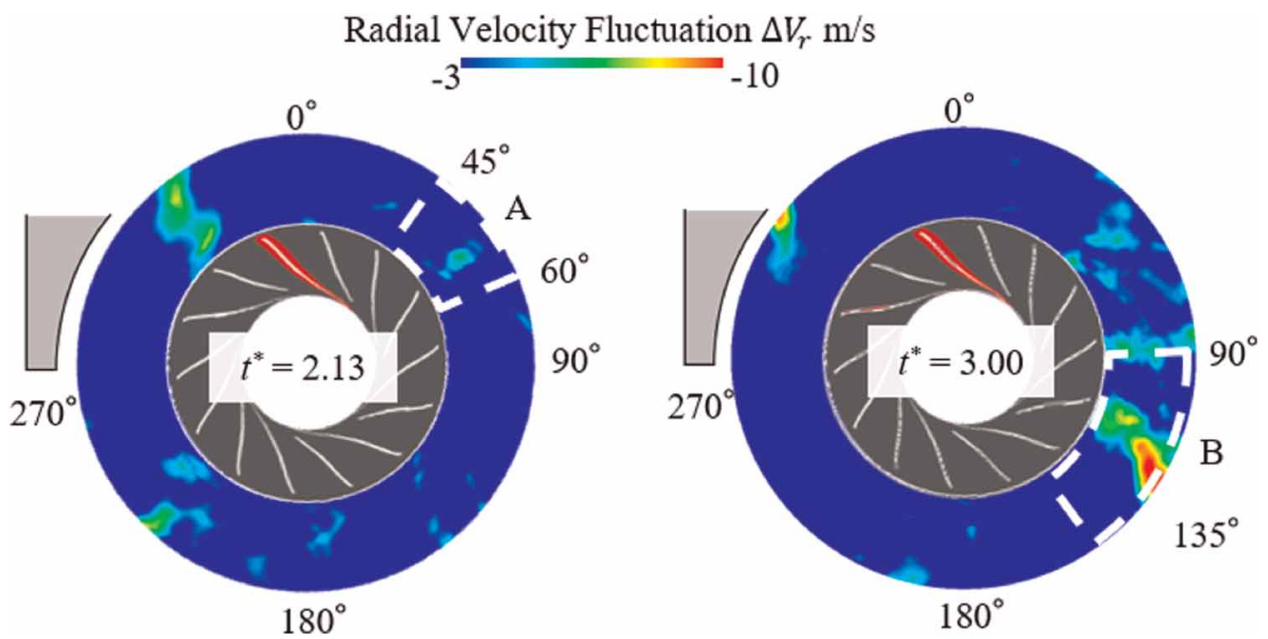

Figure 11. Instantaneous Radial Velocity Fluctuation at $t^{*}=2.13$ and 3.00 (CFD: $\phi=0.08$ ).

was investigated from Figures 12 and 13. Figures 12(i) and 13(i) show the meridional radial velocity distribution around the region $\mathrm{A}$ and $\mathrm{B}$ at $t^{*}=2.13$ and 3.00. Figures 12(ii) and 13(ii) show the iso-surface of $V_{r}=-10 \mathrm{~m} / \mathrm{s}$ colored by the distance from the hub wall. From Figure 12, the hub wall boundary layer separation initiated at around $45^{\circ}$. Then, this hub wall separation induced the next shroud wall separation because of a spanwise pressure gradient, as shown in Figure 13. The boundary layer separations on the shroud wall developed, as it can be seen by the low velocity region at diffuser exit. The hub side Backflow Region I was the impeller wake. From numerical results, the impeller wake mainly expanded on the hub side from $90^{\circ}$ to the cutoff. The shroud side Backflow Region II consisted of the boundary layer separations. The region indicated by the black circle was the low velocity region. Backflow Region I rotated faster than Backflow Region II, as shown in Figure 13a and b. Therefore, Region I caught up with Region II and induced the boundary layer separation enlargement on the shroud wall (Region II). Furthermore, the shroud wall boundary layer separations (Region II) induced the next hub wall boundary layer separations (Region III) because of a spanwise pressure gradient. The low velocity region within vaneless diffuser developed due to the boundary layer separations occurring on the shroud and hub wall by turns. Finally, the low velocity region formed the entire diffuser passage span blockage.

In order to validate the numerical results, the radial velocity fluctuation of impeller discharge flow was measured at the meridional direction (D.I.150, D.M.150 and D.E.150), as shown in Figure 14. The diffuser stall fluctuations were observed near hub side at D.I.150. On the other hand, the region with large magnitudes of the diffuser stall was shifted to the shroud side at D.M.150 and D.E.150. It is because the diffuser stall was consisted of the boundary layer separations occurring on the shroud and hub wall by turns. Therefore, the boundary layer separations occurring on the shroud and hub wall were considered as the key factors contributing to the diffuser stall generation by both experimental and CFD results. In our future work, the general generation mechanism of
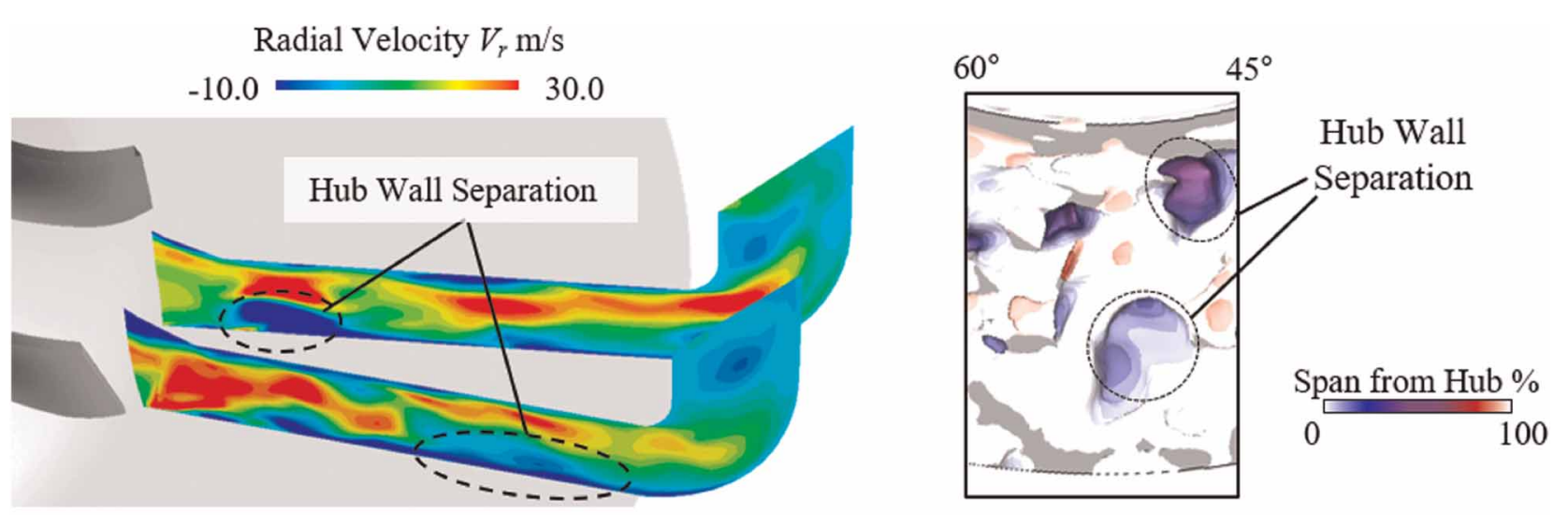

(i) Meridional radial velocity distribution

(ii) Iso-surface of back flow region

Figure 12. Generation Mechanism of Diffuser Stall Cell at $t^{*}=2.13$ (CFD: $\phi=0.08$ ). 

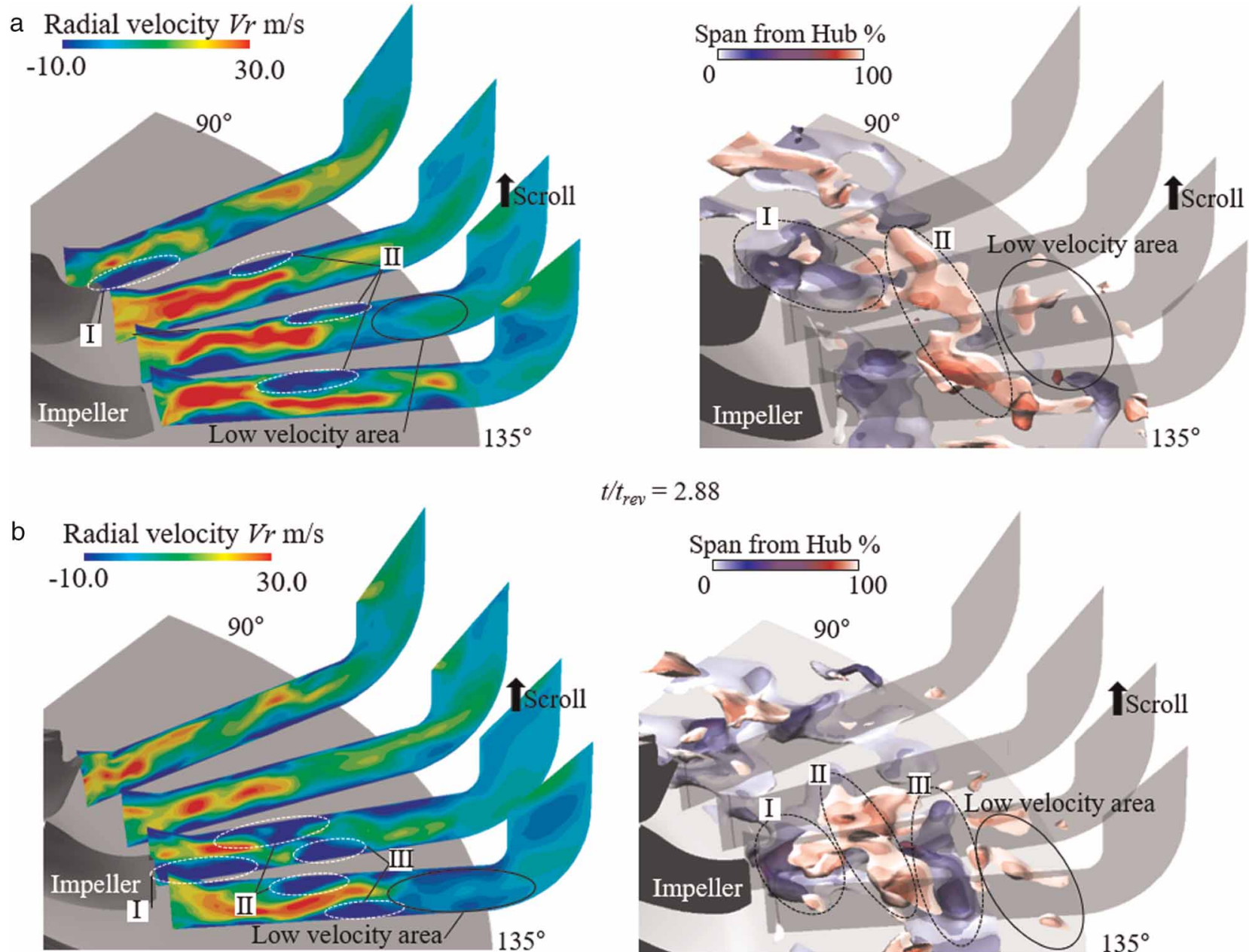

$t / t_{\text {rev }}=2.88$

(i) Meridional radial velocity distribution

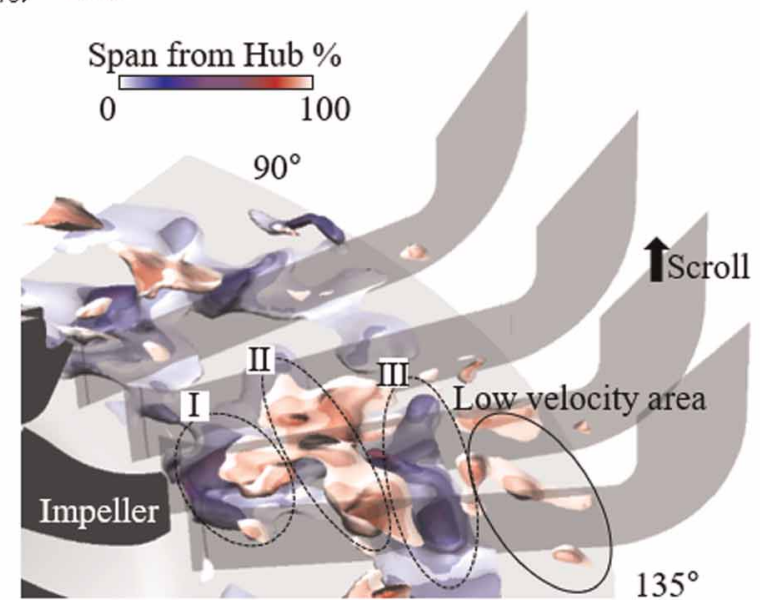

(ii) Iso-surface of back flow region

$t / t_{\text {rev }}=3.00$

Figure 13. Development of Diffuser Stall Cell at $t^{*}=2.88$ and 3.00 (CFD: $\phi=0.08$ ).

the boundary layer separations, which occurred at the hub and then the shroud end wall, will be revealed by several CFD investigations.

\section{Conclusions}

The generation mechanism of a diffuser rotating stall in a centrifugal compressor with a vaneless diffuser was investigated by experiments and CFD analysis. The results can be summarized as follows:

1. In the test compressor, fluctuations occurred at around $25-30 \mathrm{~Hz}$, were caused by diffuser stall at off design point $\phi=0.08$. The fluctuation magnitude varied depending on circumferential position with the largest fluctuations observed near the cutoff.

2. The diffuser stall behavior varied depending on circumferential position. First, the boundary layer separations initiated on the hub wall at around $45^{\circ}$ where a large circumferential adverse pressure gradient magnitude was observed. Next, the hub side separations induced the next shroud wall boundary layer separations. The shroud side boundary layer separations developed and induced a low velocity region located at the diffuser stall cell center.

3. The diffuser stall cell developed further as it approached the cutoff. The boundary layer separations developed on both shroud and hub walls by turns due to the impeller wake interaction that developed on the hub side. The boundary layer separations occurring on the shroud and hub wall were considered as the key factors contributing to the diffuser stall generation by both experimental and CFD results. 

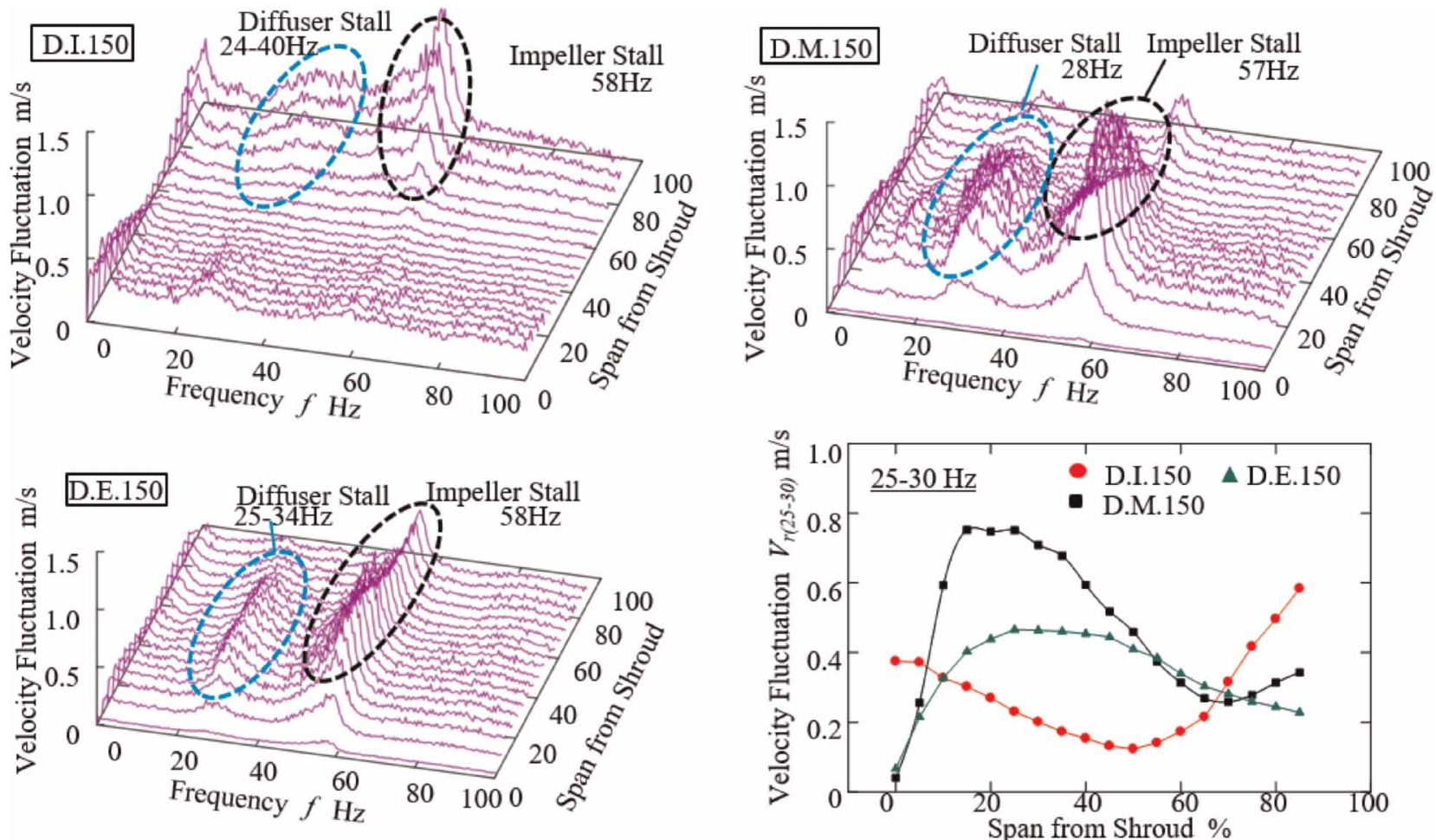

Figure 14. Distributions of Velocity Fluctuation in Meridional Direction (EXP: $\boldsymbol{\phi}=0.08$ ).

\section{Nomenclature}

$B$ diffuser passage height (m)

$D$ diameter (m)

$G$ mass flow rate $(\mathrm{kg} / \mathrm{s})$

$N$ rotational speed $\left(\mathrm{min}^{-1}\right)$

$Q$ volume flow rate $\left(\mathrm{m}^{3} / \mathrm{s}\right)$

$V_{r}$ radial velocity $(\mathrm{m} / \mathrm{s})$

$p$ static pressure $(\mathrm{Pa})$

$p_{t}$ total pressure $(\mathrm{Pa})$

$t$ time (s)

$t_{\text {rev }}$ one impeller revolution time (s)

$t^{*}$ non-dimensional time $\left(=t / t_{\text {rev }}\right)$

$Z$ number of impeller blades

$\rho$ air density $\left(\mathrm{kg} / \mathrm{m}^{3}\right)$

$\phi$ flow coefficient

$\psi_{t}$ total pressure rise coefficient

1 impeller inlet

2 impeller outlet

3 diffuser inlet

4 diffuser outlet

\section{Funding sources}

Grant-in-Aid for Early-Career Scientists No.19K14894.

\section{Competing interests}

Nobumichi Fujisawa declares that he has no conflict of interest. Kenta Tajima declares that he has no conflict of interest. Hiroshi Miida declares that he has no conflict of interest. Yutaka Ohta declares that he has no conflict of interest. 


\section{References}

Fujisawa N. and Ohta Y. (2017). Transition process from diffuser stall to stage stall in a centrifugal compressor with a vaned diffuser. International Journal of Rotating Machinery. 2861257. https://doi.org/10.1155/2017/2861257

Fujisawa N., Ikezu S., and Ohta Y. (2016). Structure of Diffuser Stall and Unsteady Vortices in a Centrifugal Compressor. ASME 2016 Turbo Expo, GT2016-56154. https://doi.org/10.1115/gt2016-56154

Fujisawa N., Inui T., and Ohta Y. (2019a). Evolution process of diffuser stall in a centrifugal compressor with vaned diffuser. ASME Journal of Turbomachinery. 141: 041009.

Fujisawa N., Takahashi M., and Ohta Y. (2019b). Transient Analysis of Rotating Stall Development in a Centrifugal Compressor with Vaned Diffuser. ASME 2019 Turbo Expo, GT2019-90797.

Im H., Chen X. Y., and Zha G. (2012). Detached-eddy simulation of rotating stall inception for a full-annulus transonic rotor. Journal of Propulsion and Power. 28 (4), 782-798. https://doi.org/10.2514/1.b34395

Iwakiri K., Furukawa M., Ibaraki S., and Tomita I. (2009). Unsteady and Three-dimensional Flow Phenomena in a Transonic Centrifugal Compressor Impeller at Rotating Stall. Proceedings of ASME Turbo Expo 2009, GT2009-59516.

Ohuchida S., Kawakubo T., and Tamaki H. (2013). Experimental Study of Rotating Stall in Vaneless Diffuser of a Centrifugal Compressor. Proceedings of ASME Turbo Expo 2013, GT2013-95468.

Senoo Y. and Kinoshita Y. (1978). Limits of Rotating Stall and Stall in Vaneless Diffuser of Centrifugal Compressors. ASME Paper, No.78-FT-19.

Senoo Y., Kinoshita Y., and Ishida M. (1977). Asymmetric flow in vaneless diffusers of centrifugal blowers. ASME Journal of Fluids Engineering. 99: 104-114. https://doi.org/10.1115/1.3448501

Strelets M. (2001). Detached Eddy Simulation of Massively Separated Flows. AIAA Paper, 2001-0879.

Van den Breambussche R. (2019). Design and analysis of centrifugal compressors: John Wiley \& Sons Ltd: ASME Press, New York, NY.

Yamada K., Furukawa M., Arai H., and Kanazaki D. (2017). Evolution of Reverse Flow in a Transonic Centrifugal Compressor at Near-surge. ASME 2017 Turbo Expo, GT2017-63568.

Zhang H., Yang C., Yang D., Wang W., Yang C., and Qi M. (2019). Investigation on the stall inception circumferential position and stall process behavior in a centrifugal compressor with volute. ASME Journal of Turbomachinery. 141: 021030.

Zhang W., Fujisawa N., Ohta Y., Goto T., and Kato D. (2020). Rotor performance of an axial flow compressor at free and highly loaded windmilling conditions. International Journal of Gas Turbine, Propulsion and Power Systems. 11 (2), 17-26. 